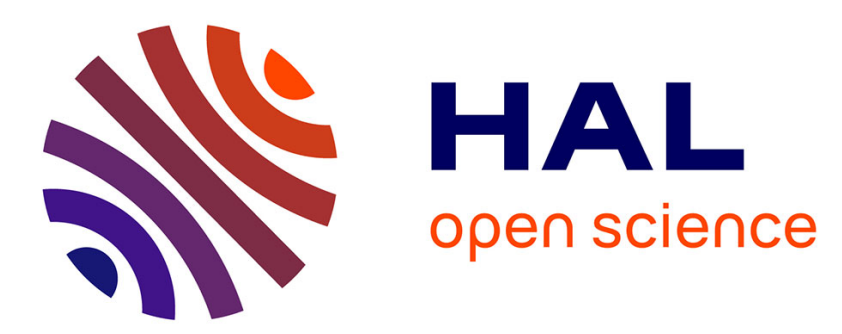

\title{
Stabilization of systems with switchings on the axis of their coordinates and its input-to-state properties
}

\author{
Andrea Aparicio, Leonid Fridman, Denis Efimov
}

\section{To cite this version:}

Andrea Aparicio, Leonid Fridman, Denis Efimov. Stabilization of systems with switchings on the axis of their coordinates and its input-to-state properties. Nonlinear Analysis: Hybrid Systems, inPress, 10.1016/j.nahs.2018.10.008 . hal-01908289

\section{HAL Id: hal-01908289 \\ https://hal.inria.fr/hal-01908289}

Submitted on 30 Oct 2018

HAL is a multi-disciplinary open access archive for the deposit and dissemination of scientific research documents, whether they are published or not. The documents may come from teaching and research institutions in France or abroad, or from public or private research centers.
L'archive ouverte pluridisciplinaire HAL, est destinée au dépôt et à la diffusion de documents scientifiques de niveau recherche, publiés ou non, émanant des établissements d'enseignement et de recherche français ou étrangers, des laboratoires publics ou privés. 


\title{
Stabilization of systems with switchings on the axis of their coordinates and its input-to-state properties
}

\author{
Andrea Aparicio $^{1 *}$, Leonid Fridman $^{1}$, Denis Efimov ${ }^{2}$ \\ ${ }^{1}$ A. Aparicio and L. Fridman are with Departamento de Ingeniería de Control y Robótica, División \\ de Ingeniería Eléctrica, Facultad de Ingeniería UNAM, Edificio T, Segundo piso Ciudad Universi- \\ taria D.F., México. \\ ${ }^{2}$ D. Efimov is with Inria, Univ. Lille, CNRS, UMR 9189 - CRIStAL, F-59000 Lille, France; and \\ Department of Control Systems and Informatics, University ITMO, 197101 Saint Petersburg, Rus- \\ sia. \\ *andrea.aparicio.martinez@gmail.com
}

\begin{abstract}
The stabilization problem for switched systems in which switchings occur on the axes of its state coordinates is considered. It is shown that a linear feedback, or a combination of linear feedback and a switching law, can be designed such that the closed-loop is stable, and has the inputto-state property, allowing to guarantee robustness against matched and unmatched perturbations. The conditions of stability are expressed in the form of linear matrix inequalities. The results are illustrated by numerical simulations.
\end{abstract}

\section{Introduction}

The study and implementation of systems that commute between different behavior schemes are strong mathematical and technical challenges. The theoretical results available are gathered in the literature of switched, hybrid, and variable structure systems; a survey on the existing stability criteria for the first can be found in [1]. The switched systems domain can be divided in two big groups: the systems whose switchings depend on the time, and those that operate under statedependent switching laws. The latter group can also be divided in two: the systems with given switching laws, that need to be stabilized in some way, or those for which the design of a stabilizing switching law is required. Numerous works can be found that offer stability results based on different well-known strategies, and the most utilized are Linear Matrix Inequalities (LMIs) and Lyapunov methods $([2,3,4])$. The state-dependent switching can be used to stabilize the system with the commutation law directly following the expression of a Lyapunov function $([5,6])$. In some cases the dwell-time restriction is imposed ([7]), then asymptotic rates of convergence can be obtained. Skipping the requirement on a minimum time between commutations it is possible to ensure a finite-time rate of convergence in the system, as in the sliding mode control framework $([8,9])$. Though in all these examples the stabilization is achieved, some perturbations might occur such that the sliding-mode is lost and the trajectories end up diverging, causing undesired or even dangerous behaviour in the related applications.

In this paper we will focus on state-dependent switching systems and, in particular, those whose switchings occur on the axes of its state coordinates, in other words, at the precise instant when a 
change on the sign of the state variables occurs (frequently, such a condition can be ensured after a proper transformation of coordinates). An example of a physical realization of this class of systems is the ones that include relay or on-off electrical components in their configuration, and the study of the stability of such systems is one of the oldest control problems $([10,11,12,13,14])$. A well-known real-world scenario of such a class of systems includes the mechanical dynamics with dry friction, when sign of the velocity determines the direction of the friction force.

The state space study of one-dimensional relay systems was performed in [15] (see also [16]). In that work it was proven that a first order relay system with relative degree one is semi-globally finite-time stable if the switches occur on the axis of its state variable. In the same work it was shown that a system of relative degree two that switches on the axis of its first coordinate is semiglobally exponentially stable if a linear stabilizer dependent on the second coordinate is added. Moreover, it was proven that a system with relative degree greater than two that switches on the axis of its state coordinates is never stable unless some linear stabilizing terms are added.

In this paper, a particular class of systems is dealt with, whose dynamics are driven by uncertain chains of integrators with the signs of all the state variables in the last coordinate. The semi-global finite-time convergence of this kind of systems, for the case of order two, was proved in [17]. Almost three decades after that, in [18] it was shown that for the third order, the majority of solutions converge to zero in finite-time, but there is also a two dimensional set of equilibrium points different from zero. This set of equilibrium points of dimension $n-1$ also appears for chain of integrators of order $n$ with $n$-relays in the dynamics of the last state variable.

The stability in the above mentioned cases is quite fragile and even a slight perturbation makes the trajectories of these systems escape to infinity. In order to ensure robustness against perturbation, the typical procedure is to design gains of a switching controller based on an upper bound of the perturbation, so it is required that this bound is known. Also, it is typically assumed that the perturbations are matched to the control input because unmatched perturbations, even when an upper bound to them is known, can also easily destroy the stability of switching systems. Some works that offer solutions to the unmatched disturbance problem are [19, 20, 21].

Considering these results, one can conclude that if the system switches every time on the axes of its state space variables, it is impossible to establish its global convergence to the origin, and then some stabilizers should appear in the system's structure, or added as controllers, in order to achieve this goal. Also, that some actions must be taken in order to ensure some robustness of the closed loop to certain perturbations, at least.

In this paper, for an arbitrary order system with switches on the axes of each of its coordinates (the switches can occur in any of the state variables), the conditions are established that allow the stability to be kept by adding a linear compensator. The resulting closed loop can be placed within the classifications 1 and 3, mentioned in the tutorial paper [22], that refer to relays with constant gains, and linear feedbacks with switched gains, respectively. Even more, we investigate de input-to-state stability (ISS) of the closed loop, to ensure certain robustness against bounded perturbations. The main contributions of the paper are

- Sufficient conditions for the linear stabilizer design.

- The ISS properties of the closed loop. 
The work is organized as follows: Section 2 includes the notation used throughout this paper, as well as some known results useful for the development of the methodology. Section 3 includes the problem statement. In Section 4 the form of the control signal is proposed, and the stability of the closed loop is proved by constructing a Lyapunov equation. The stability conditions are presented in the form of LMIs. In Section 4.1 the input-to-state stability property of the closed loop system is investigated, which guarantees robustness to external, unknown, bounded inputs that can be both matched and unmatched. Academic examples are provided in Section 5 along with some numerical simulations that validate the theoretical results. Finally, Section 6 contains some conclusions of this work.

\section{Preliminaries}

\subsection{Notation}

- The element-wise application of an operator $\bullet$ to a vector $v$ is indicated by $\vec{\bullet}(v)$;

- $I_{n}$ denotes the Identity matrix of dimension $n$;

- $\operatorname{diag}(A)$ represents a matrix where the main diagonal is the same as that of matrix $A$ and every other element is equal to zero;

- $\vec{a}_{(n \times s)}$, for a constant $a$ represents a matrix of dimension $n \times s$, whose every element is equal to $a$;

- $A_{i j}$ represents the element in the position $(i, j)$ of a matrix $A$;

- $A^{[i]}$ denotes the $i$ th column of a matrix $A$;

- $A_{n}^{\text {int }}$ represents a square matrix of size $n$ whose every element is equal to zero, except for the diagonal above the main one, which is composed of ones;

- $\lambda_{\min }(A)$ and $\lambda_{\max }(A)$ represent the minimal and the maximal eigenvalues of a matrix $A$, respectively;

- $|a|$ represents the absolute value of a scalar $a$;

- $\|v\|$ denotes the Euclidean norm of a vector $v \in \mathbb{R}^{n},\|v\|_{1}=\sum_{i=1}^{n}\left|v_{i}\right|$ and $\|v\|_{\infty}=$ $\max _{1 \leq i \leq n}\left|v_{i}\right|$

- $\|A\|$ represents the induced norm of a matrix $A \in \mathbb{R}^{n \times s}$, while $\|A\|_{1}=\max _{1 \leq i \leq s}\left\|A^{[i]}\right\|_{1}$ and $\|A\|_{\infty}=\left\|A^{T}\right\|_{1}$

- For a matrix $A \in \mathbb{R}^{n \times s}$ the following norm equivalences hold ([23])

$$
\frac{1}{\sqrt{n}}\|A\|_{1} \leq\|A\| \leq \sqrt{s}\|A\|_{1} .
$$

For $a$ and $b$ nonnegative real numbers, and if $p$ and $q$ are some positive real numbers such that $\frac{1}{p}+\frac{1}{q}=1$ the following inequality holds ([24])

$$
a b \leq \frac{a^{p}}{p}+\frac{b^{q}}{q}
$$


- The set of all functions endowed with the (essential) supremum norm $\|\omega\|_{\infty}=($ ess $) \sup _{t \geq 0}\|\omega(t)\|<$ $\infty$, is denoted by $L_{\infty}^{m}$

Definition 1. ISS ([25]): A system $\dot{x}=f(x, u)$ is globally input-to-state stable (ISS) if there exists a $\mathcal{K} \mathcal{L}$-function $\beta: \mathbb{R}_{\geq 0} \times \mathbb{R}_{\geq 0} \rightarrow \mathbb{R}$ and a $\mathcal{K}$-function $\gamma: \mathbb{R}_{\geq 0} \rightarrow \mathbb{R}_{\geq 0}$ such that, for each input $\omega \in L_{\infty}^{m}$ and each initial state $x(0) \in \mathbb{R}^{n}$, it holds, for each $t \geq 0$ that

$$
\|x(t, x(0), u)\| \leq \beta(\|x(0)\|, t)+\gamma\left(\|\omega\|_{\infty}\right)
$$

Definition 2. ISS-Lyapunov function ([25]): A smooth function $V: \mathbb{R}^{n} \rightarrow \mathbb{R}_{\geq 0}$ is called ISSLyapunov function for a system $\dot{x}=f(x, u)$ if there exist $\mathcal{K}_{\infty}$-functions $\alpha_{1}, \alpha_{2}$, and $\mathcal{K}$-functions $\alpha_{3}$ and $\chi$, such that

$$
\alpha_{1}(\|x\|) \leq V(x) \leq \alpha_{2}(\|x\|)
$$

for any $x \in \mathbb{R}^{n}$, and

$$
\nabla V(x) f(x, \omega) \leq-\alpha_{3}(\|x\|)
$$

for any $x \in \mathbb{R}^{n}$ and any $\omega \in \mathbb{R}^{m}$ such that $\|x\| \geq \chi(\|\omega\|)$.

Theorem 1. Nonsmooth Lyapunov Functons (Theorem 3.1 in [26]) Suppose that in a domain $\left(x \in \mathcal{B}_{\delta}, t \in \mathbb{R}\right)$, where $\mathcal{B}_{\delta}$ is a ball centred at the origin with radius $\delta>0$, there exists a Lipschitz continuous, positive definite, decrescent function $V(x, t)$ such that its time derivative, computed along the trajectories $x(t)$ of the discontinuous system $\dot{x}=f(x, t)$, which are initialized within $\mathcal{B}_{\delta}$, is negative semidefinite almost everywhere for almost all $t$, then $\dot{x}=f(x, t)$ is uniformly stable.

\section{Problem Statement}

Consider a system in the form

$$
\begin{aligned}
& \dot{x}=\bar{A}_{0} x+\bar{A}_{1} \operatorname{sign}(x)+B u+\omega \\
& y=x,
\end{aligned}
$$

where $x \in \mathbb{R}^{n}$ is the state vector, $\overrightarrow{\operatorname{sign}}(x) \in \mathbb{R}^{n}$ is a column defined as $\overrightarrow{\operatorname{sign}}^{T}(x):=\left[\begin{array}{lll}\operatorname{sign}\left(x_{1}\right) & \ldots & \operatorname{sign}\left(x_{n}\right)\end{array}\right]$, $u \in \mathbb{R}^{m}$, with $m \leq n$ is the control input, and $\omega \in \mathbb{R}^{p}$ is a vector that contains all the perturbations or external, unknown inputs. Here, $p \leq n$. The terms $\bar{A}_{0}, \bar{A}_{1} \in \mathbb{R}^{n \times n}$, and $B \in \mathbb{R}^{n \times m}$ are known, real and constant matrices. Since sliding modes may occur, all the solutions are understood in the sense of Filippov ([27]).

In its most general form, system (2) consists of a linear part and a part that switches on the axis of the states $x$. The perturbations are assumed bounded, that is $\|\omega\| \leq \bar{\omega}$, but $\bar{\omega}$ is assumed unknown. Also, there is no restriction to the matchedness of the perturbations to the control input. The goal is to design a control $u$ that takes the trajectories of the system to the origin, robustly in spite of the perturbations.

\section{Control Design and Stability Analysis}

In this section the nominal case of (2) will be considered. That is

$$
\begin{aligned}
\dot{x} & =\bar{A}_{0} x+\bar{A}_{1} \operatorname{sign}(x)+B u \\
y & =x,
\end{aligned}
$$


Now, let the control signal $u$ be

$$
u=K_{0} x+K_{1} \operatorname{sign}(x)
$$

where $K_{0}, K_{1} \in \mathbb{R}^{(n \times n)}$ (see footnote ${ }^{1}$ ). In the rest of the section a stability proof for the closed loop

$$
\dot{x}=A_{0} x+A_{1} \operatorname{sign}(x)
$$

where $A_{0}=\left(\bar{A}_{0}+B K_{0}\right)$, and $A_{1}=\left(\bar{A}_{1}+B K_{1}\right)$ will be provided, along with some conditions for the gain matrices $K_{0}$ and $K_{1}$. This will be performed by establishing a sufficient LMI condition to construct a Lyapunov function for (4). To this end, the matrices $P \in \mathbb{R}^{n \times n}, G \in \mathbb{R}^{n \times n}, R \in \mathbb{R}^{n \times n}$, $r \in \mathbb{R}^{n \times n}$ and $M \in \mathbb{R}^{n \times n}$, and a constant $\mu$ must be defined in the following manner:

$$
\begin{gathered}
P:=\left[\begin{array}{cccc}
p_{11} & p_{12} & \ldots & p_{1 n} \\
p_{12} & p_{22} & \ldots & p_{2 n} \\
\vdots & \ddots & \ddots & \vdots \\
p_{1 n} & p_{2 n} & \ldots & p_{n n}
\end{array}\right], \quad G:=\left[\begin{array}{cccc}
g_{1} & 0 & \ldots & 0 \\
0 & \ddots & \ddots & \vdots \\
\vdots & \ddots & \ddots & 0 \\
0 & \ldots & 0 & g_{n}
\end{array}\right] \\
R:=2 A_{1}^{T} P+G A_{0}, \quad R_{d}:=\operatorname{diag}(R), \quad M:=R_{d}+\left|R-R_{d}\right|,
\end{gathered}
$$

for some real constants $g_{i}, p_{i j}, i, j=1, \ldots, n$. Note that in the definition of $M$ the absolute value of each element of the resulting matrix of the subtraction should be considered, and not any norm.

Theorem 2. Let the origin be the only equilibrium of (4), and the following pair of LMIs be satisfied

$$
A_{0}^{T} P+P A_{0}=-Q, \quad M \overrightarrow{1}_{(n \times 1)} \stackrel{\overrightarrow{\leq} 0}{0}
$$

for

$$
P=P^{T}>0, \quad Q=Q^{T}>0, \quad G \geq 0, \quad G A_{1}=0 .
$$

Then, a function $V: \mathbb{R}^{n} \rightarrow \mathbb{R}$ defined as

$$
V(x):=x^{T} P x+\sum_{i=1}^{n} g_{i}\left|x_{i}\right|
$$

is a Lyapunov function for system (4), with a derivative estimate

$$
\dot{V} \leq-x^{T} Q x .
$$

Proof. Since the matrix $P$ is positive definite and $G$ is positive semidefinite, then $V$ is also positive definite and radially unbounded. The function $V$ is locally Lipschitz continuous. Then, by Rademacher's theorem ([28]), it is differentiable almost everywhere in $\mathbb{R}^{n}$, and since $G A_{1}=0$, the derivative of $V$ along the trajectories of (4) is

$$
\begin{aligned}
\dot{V} & =x^{T}\left(A_{0}^{T} P+P A_{0}\right) x+\overrightarrow{\operatorname{sign}}^{T}(x)\left(A_{1}^{T} P+\frac{1}{2} G A_{0}\right) x+x^{T}\left(P A_{1}+\frac{1}{2} A_{0}^{T} G\right) \operatorname{sign}(x) \\
& =x^{T}\left(A_{0}^{T} P+P A_{0}\right) x+\operatorname{sign}(x)^{T} R x .
\end{aligned}
$$

\footnotetext{
${ }^{1}$ In Section 3 it was mentioned that the system (2) in its most general form consists of a linear part plus a switched part and it is implied that one of these parts might not be present. It is the same case for the control signal, which could be only a linear control, a switched one, or a combination of both, the restriction being that the closed loop has the form (4), for the nominal case.
} 
The Lyapunov equation $A_{0}^{T} P+P A_{0}=-Q$ can be solved for $P=P^{T}>0$, with $Q=Q^{T}>0$ if and only if $A_{0}$ is a stable matrix. From the second part of (7) we have that

$$
\begin{aligned}
\operatorname{sign}(x)^{T} R x & =\sum_{i=1}^{n}\left[x_{i} \sum_{k=1}^{n}\left(\operatorname{sign}\left(x_{k}\right) R_{k, i}\right)\right] \\
& \leq \sum_{i=1}^{n}\left[\left(R_{i, i}+\sum_{k \neq i, k=1}^{n}\left|R_{k, i}\right|\right)\left|x_{i}\right|\right]
\end{aligned}
$$

and with $M$ as defined above, we get

$$
R_{i, i}+\sum_{k \neq i}^{n}\left|R_{k, i}\right| \leq 0, \forall 1 \leq i \leq n \Leftrightarrow M^{T} \overrightarrow{1}_{(n \times 1)} \overrightarrow{\leq} 0 .
$$

Thus,

$$
\dot{V} \leq-x^{T} Q x
$$

and if the conditions of Theorem 2 are satisfied, the function $V$ is positive definite for all $x$, its derivative along the trajectories of (4) is negative definite, and by Theorem 1, (4) is uniformly stable.

Remark 1. The restrictions imposed on the matrix $M$ can be replaced with the condition that

$$
\mu_{1}(R) \leq 0
$$

where $\mu_{1}(R)=\sup _{i} R_{i, i}+\sum_{k \neq i}\left|R_{k, i}\right|$ is the logarithmic norm of the matrix $R$ calculated for the norm $|\cdot|_{1}$.

\subsection{Perturbation Analysis and Input to State Stability}

In this section we will prove that if system (4) satisfies the conditions of Theorem 2, it also admits the ISS property, which makes it robust to exogenous matched or unmatched bounded perturbations. It is important to note that while the boundedness of the external inputs is a fundamental condition for establishing the ISS property, the knowledge of its supremun norm is not necessary for the design. Consider system (4) with an unknown input function $\omega: \mathbb{R}_{\geq 0} \rightarrow \mathbb{R}^{n}$ :

$$
\dot{x}=A_{0} x+A_{1} \operatorname{sign}(x)+\omega .
$$

Theorem 3. If $\omega$ is essentially bounded, i.e. $\omega \in L_{\infty}$, and the conditions of Theorem 2 are satisfied, then (8) is ISS with an asymptotic gain $\gamma: \mathbb{R}_{\geq 0} \rightarrow \mathbb{R}_{\geq 0}$ given by

$$
\gamma(r)=\alpha_{1}^{-1} \circ \alpha_{2} \circ \alpha_{3}^{-1} \circ \alpha_{4}(r),
$$

where for any $\epsilon_{1} \in\left(0, \frac{\lambda_{\min }(Q)}{\lambda_{\max }(P)}\right)$,

$$
\begin{aligned}
\alpha_{1}(r) & :=\lambda_{\min }(P) r^{2} \\
\alpha_{2}(r) & :=\left(\lambda_{\max }(P) r+\sqrt{n} \max \left\{g_{i}\right\}\right) r \\
\alpha_{3}(r) & :=\left(\lambda_{\min }(Q)-\epsilon_{1} \lambda_{\max }(P)\right) r \\
\alpha_{4}(r) & :=\left(\frac{\lambda_{\max }(P)}{\epsilon_{1}} r+\sqrt{n} \max \left\{g_{i}\right\}\right) r .
\end{aligned}
$$


Note that the magnitude of the gain $\gamma(r)$ is proportional to the square of $r$, and that the established result is of qualitative nature and that quantitatively they would only represent the worst possible scenario.

Proof. The derivative of (6) over the trajectories of (8) is

$$
\dot{V}=-x^{T} Q x+\dot{\operatorname{sign}}^{T}(x) R x+2 x^{T} P \omega+\operatorname{sign}^{T}(x) G \omega .
$$

For the above expression the following inequalities hold (see references in Section 2):

$$
\begin{aligned}
\operatorname{sign}^{T}(x) R x & \leq 0 \\
-x^{T} Q x & \leq-\lambda_{\min }(Q)\|x\|^{2} \\
2 x^{T} P \omega & \leq \lambda_{\max }(P)\left(\epsilon_{1}\|x\|^{2}+\frac{\|\omega\|^{2}}{\epsilon_{1}}\right) \forall \epsilon_{1}>0 \\
\operatorname{sign}^{T}(x) G \omega & \leq \sqrt{n} \max \left\{g_{i}\right\}\|\omega\|, i=1, \ldots, n .
\end{aligned}
$$

From definition 2, $V$ is an ISS-Lyapunov function for (8) with $\chi(r)=\alpha_{3}^{-1} \circ \alpha_{4}(r)$, since

$$
\begin{gathered}
\alpha_{1}(\|x\|) \leq V(x) \leq \alpha_{2}(\|x\|), \\
\dot{V} \leq-\alpha_{3}(\|x\|)+\alpha_{4}(\|\omega\|)
\end{gathered}
$$

with $\alpha_{1}, \alpha_{2}, \alpha_{3}, \alpha_{4} \in \mathcal{K}_{\infty}$, as defined in the theorem. Then, following the arguments of [29], the system is ISS and the asymptotic gain function is $\gamma(r)$.

\section{Examples and numerical simulations}

All simulations have been performed in Matlab software using the explicit Euler method with the discretizations step 0.001 .

\subsection{Interconnected mechanical systems}

Consider two interconnected mechanical systems whose velocities are given by variables $x_{1}$ and $x_{2}$ respectively, and suppose that the acceleration of each of them can be controlled. The state space representation of this system can be written as

$$
\begin{aligned}
{\left[\begin{array}{l}
\dot{x}_{1} \\
\dot{x}_{2}
\end{array}\right] } & =\left[\begin{array}{ll}
a_{11} & a_{12} \\
a_{21} & a_{22}
\end{array}\right]\left[\begin{array}{l}
x_{1} \\
x_{2}
\end{array}\right]+\left[\begin{array}{l}
\omega_{1} \\
\omega_{2}
\end{array}\right]+\left[\begin{array}{l}
u_{1} \\
u_{2}
\end{array}\right] \\
y & =\left[\begin{array}{ll}
1 & 0 \\
0 & 1
\end{array}\right]\left[\begin{array}{l}
x_{1} \\
x_{2}
\end{array}\right]
\end{aligned}
$$

System (11) is linear, and the switches will be added as control signals. Two perturbations are considered, $\omega_{1} \in \mathbb{R}$ and $\omega_{2} \in \mathbb{R}$, one on each of the systems. These perturbations are assumed to be bounded, but the value of their supremum norms is considered unknown. Suppose that the parameters of (11) are $a_{11}=-1, a_{12}=0.5, a_{21}=0.5$ and $a_{22}=-1$. If the control signals are chosen as

$$
u_{1}=-2 \operatorname{sign}\left(x_{1}\right)-\operatorname{sign}\left(x_{2}\right) \quad \text { and } \quad u_{2}=-\operatorname{sign}\left(x_{1}\right)-2 \operatorname{sign}\left(x_{2}\right),
$$


then (11) can be written in the form (4) with

$$
A_{0}=\left[\begin{array}{ll}
-1 & 0.5 \\
0.5 & -1
\end{array}\right], \quad \text { and } \quad A_{1}=\left[\begin{array}{ll}
-2 & -1 \\
-1 & -2
\end{array}\right]
$$

Matrix $A_{0}$ is Hurwitz, so the Lyapunov equation can be satisfied for a positive definite matrix $P \in$ $\mathbb{R}^{(2 \times 2)}$, with $Q=I_{2}$. Choosing $g_{1}=g_{2}=0$, yields the product $M\left[\begin{array}{ll}1 & 1\end{array}\right]^{\top}=\left[\begin{array}{ll}-0.67 & -0.67\end{array}\right]^{\top}$, whose every element is clearly negative. The conditions of Theorem 2 are satisfied. The lefthand side of Figure 1 shows the simulation results of the closed loop of (11) with (12), for the unperturbed case (upper-left), and when the perturbations are chosen as $\omega_{1}=1+0.5 \sin (t)$ and $\omega_{2}=1.5+1.5 \cos (t)$ (lower-left). Note that the value of these perturbations is not taken into account for the design. For comparison purposes, the right-hand side of Figure 1 shows the simulations results of the same system (11) with and without perturbations, in closed loop with a linear feedback

$$
\left[\begin{array}{l}
u_{1} \\
u_{2}
\end{array}\right]=\left[\begin{array}{l}
-2 x_{1}-x_{2} \\
-x_{1}-2 x_{2}
\end{array}\right] .
$$

The theoretical results are validated through the simulations, which show that the trajectories of
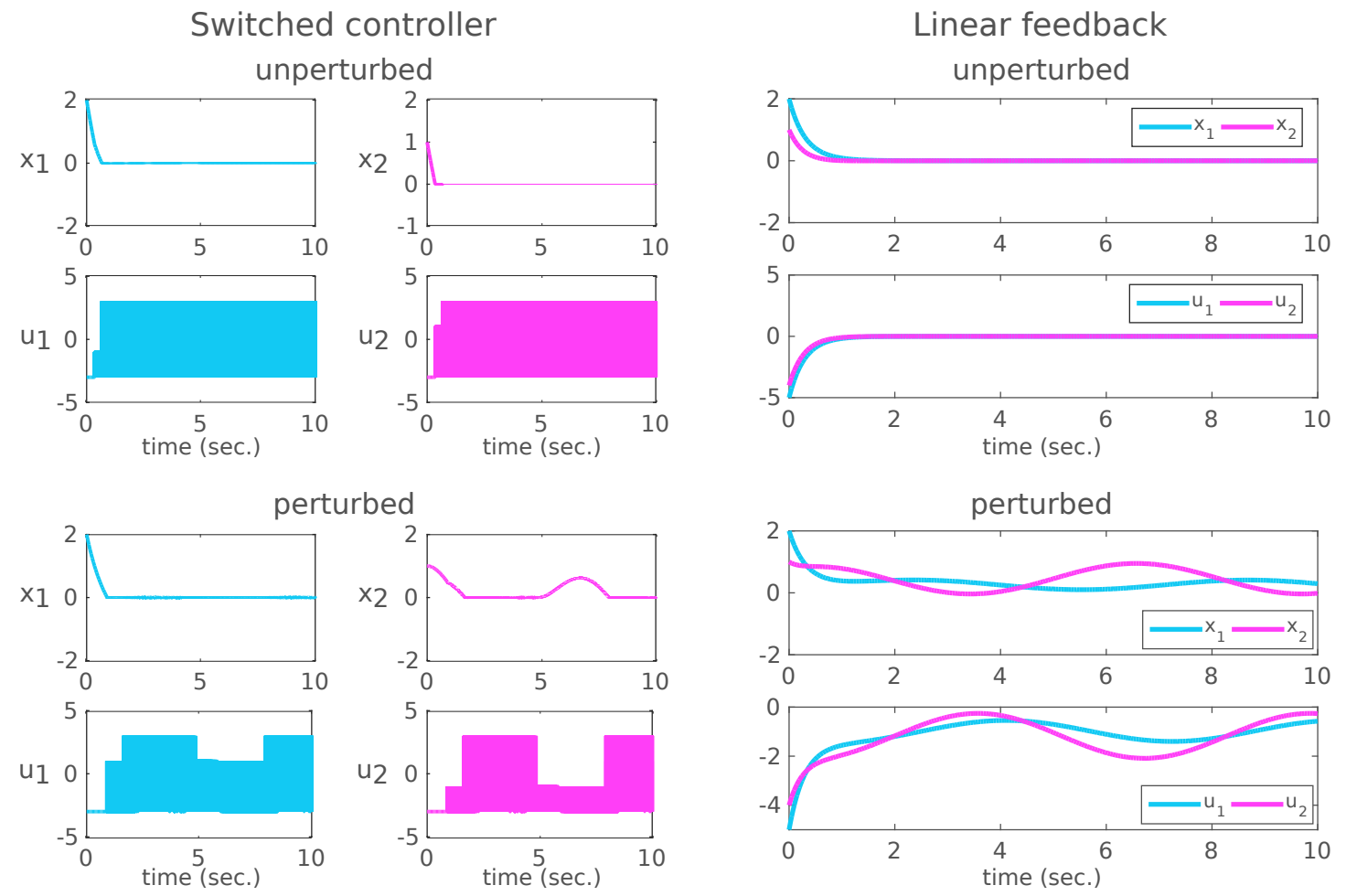

Fig. 1. Simulations results of system (11) with control (12), and with control (13).

the system converge to the origin for the unperturbed case, and to a vicinity of the origin when the perturbations are present. Note that for the case when the sign controller is added to the perturbed system, the sliding mode is lost occasionally, but the trajectories remain bounded and then return to the sliding motion. 


\subsection{Third order system}

Consider a third order system in the form (2) with

$$
A_{0}=\left[\begin{array}{lll}
0 & 1 & 0 \\
0 & 0 & 1 \\
0 & 0 & 0
\end{array}\right], \quad \bar{A}_{1}=\left[\begin{array}{ccc}
0 & 0 & 0 \\
0 & 0 & 0 \\
-2 & -2 & -2.5
\end{array}\right], \quad B=\left[\begin{array}{l}
1 \\
1 \\
1
\end{array}\right]
$$

where the initial conditions are given by $x_{0}^{T}=\left[\begin{array}{lll}x_{1}(0) & x_{2}(0) & x_{3}(0)\end{array}\right]$. In [18] it is proven that this system, for initial conditions that belong to a set $\mathcal{S}$ defined in that same work, converge to an equilibrium point different to the origin, as is shown in a) of Figure 2, for

$$
x_{0}^{T}=\left[\begin{array}{lll}
5 & -5 & 5
\end{array}\right] \in \mathcal{S} \text {. }
$$

Moreover, when a perturbation $\omega^{\top}=\left[\begin{array}{lll}2+2 \sin (t) & 0 & 0\end{array}\right]$, the trajectories diverge from the origin, as is shown in b) of Figure 2. If a control law is chosen as
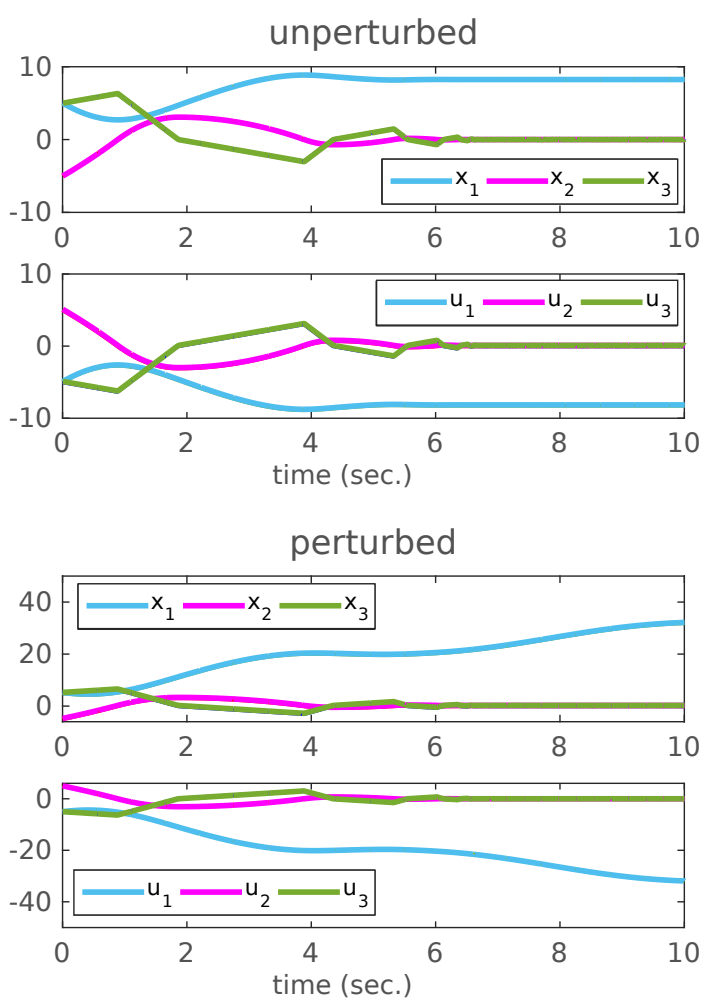

Fig. 2. State trajectories of (2) with initial conditions (15) and parameters (14) a) without perturbations, $b$ ) with perturbation $\omega$.

$$
u=-I_{3} x,
$$

the closed loop satisfies the conditions of Theorem 2, and the trajectories converge to the origin with $\omega=0$ as shown in a) of Figure 3, and to a neighborhood of the origin when $\omega^{\top}=$ $\left[\begin{array}{lll}2+2 \sin (t) & 0 & 0\end{array}\right]$ as shown in b) of Figure 3, which confirms the result of Theorem 3. Note that in this case, the addition of the linear controller prevents the trajectories of the system to escape to infinity in the perturbed case, as opposed to what happens in the uncontrolled case. This is the direct consequence of having achieved an ISS behaviour through the linear control, which is impossible to achieve with a purely switched system. 

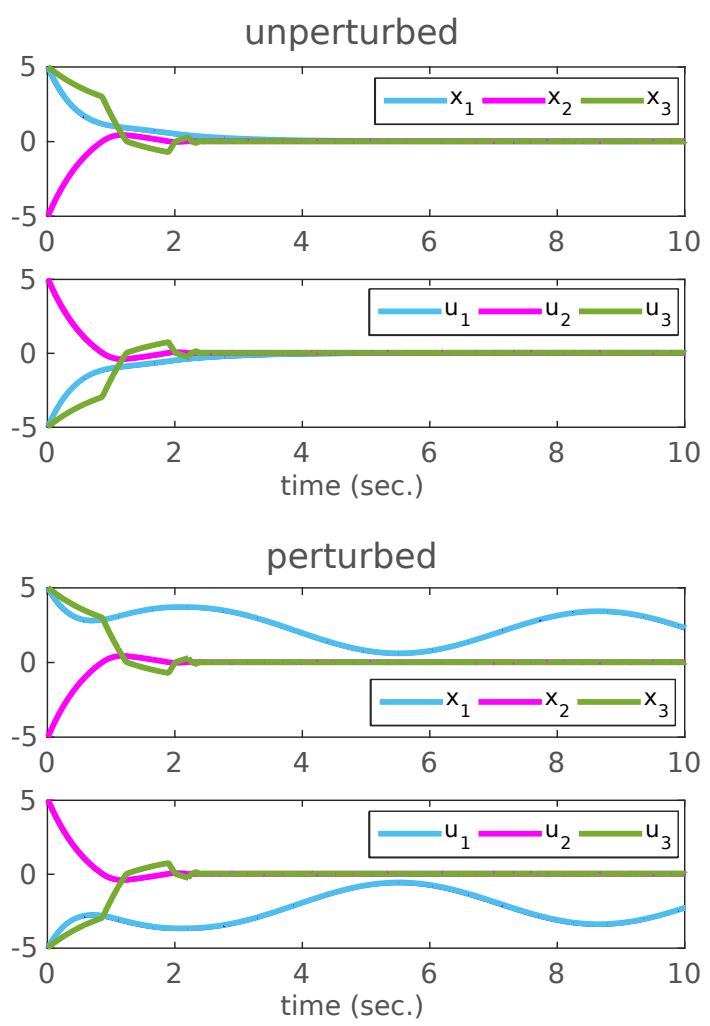

Fig. 3. State trajectories of (2) with initial conditions (15) and parameters (14) a) without perturbations, $b$ ) with perturbation $\omega$.

\section{Conclusions}

The stabilization problem of a class of switched systems, in which the switchings occur on the axes the state coordinates is considered. Sufficient condition for the design of a linear stabilizer guaranteeing the convergence of the solutions to the origin are found. The stability proof of the closed loop is performed by means of Linear Matrix Inequalities. Moreover, the ISS property has been quantified, guaranteeing robustness against both matched and unmatched perturbations, and ensuring that the trajectories of the system will remain bounded no matter how large the present perturbation is, provided that it is bounded as well. Using the presented method, for a system whose dynamics are driven by a combination of a linear part and the signs of its state variables, a stabilizing linear feedback, a switching law, or a combination of both can be designed such that the stability of the closed loop can be checked, and its ISS properties established. Some numeric examples are provided validating the theoretical results. In particular, it is shown that for the case of a chain of three integrators, with the signs of its state variables on the last coordinate, the trajectories converge to the origin, and not to another equilibrium point, when a linear stabilizer satisfying the conditions of our main theorem is added. Moreover, it is shown that the addition of a perturbation that causes the uncontrolled trajectories to escape to infinity, do not destroy the stability of the closed loop, and that its trajectories always remain bounded. As directions of future research, the control gain tuning rules development and the robustness margin optimization can be indicated, possibly by solving an optimization problem to choose the constants of the controller matrices, as well as the matrices of the Lyapunov function. 


\section{Acknowledgment}

This work was supported in part by CONACyT CVU 335111; by the Government of Russian Federation (Grant 08-08) and the Ministry of Education and Science of Russian Federation (Project 14.Z50.31.0031); by Programa de Apoyo a Proyectos de Investigación e Innovación Tecnológica (UNAM) 113216 and by HoTSMoCE INRIA associate team program.

\section{References}

[1] R. Shorten, F. Wirth, O. Mason, K. Wulff, and C. King, "Stability criteria for switched and hybrid systems," SIAM Review, vol. 49, no. 4, pp. 545-592, 2007.

[2] S. Pettersson and B. Lennartson, "Lmi for stability and robustness of hybrid systems," in Proceedings of the 1997 American Control Conference (Cat. No.97CH36041), vol. 3, Jun 1997, pp. 1714-1718 vol.3.

[3] M. Johansson and A. Rantzer, "Computation of piecewise quadratic lyapunov functions for hybrid systems," IEEE Transactions on Automatic Control, vol. 43, no. 4, pp. 555-559, Apr 1998.

[4] M. A. Wicks, P. Peleties, and R. A. DeCarlo, "Construction of piecewise lyapunov functions for stabilizing switched systems," in Proceedings of 1994 33rd IEEE Conference on Decision and Control, vol. 4, Dec 1994, pp. 3492-3497 vol.4.

[5] L. Hetel and E. Bernuau, "Local stabilization of switched affine systems," IEEE Transactions on Automatic Control, vol. 60, no. 4, pp. 1158-1163, April 2015.

[6] K. Zohra, F. Christophe, H. Laurentiu, and B. Lotfi, "Stabilization of switched affine systems with disturbed statedependent switching laws," International Journal of Robust and Nonlinear Control, vol. 28, no. 2, pp. 582-595, 2017.

[7] L. I. Allerhand and U. Shaked, "Robust state-dependent switching of linear systems with dwell time," IEEE Transactions on Automatic Control, vol. 58, no. 4, pp. 994-1001, April 2013.

[8] V. Utkin, J. Guldner, and J. Shi, Sliding Mode Control in Electro-Mechanical Systems. Taylor and Francis CRC Press, 2009.

[9] Y. Shtessel, C. Edwards, L. Fridman, and A. Levant, Sliding Mode Control and Observation. Birkhauser Basel, 2014.

[10] I. Flugge-Lotz, Discontinuous Automatic System. New York: Princeton University Press, 1953.

[11] V. Kulebakin, On Theory of Vibration Controller for Electric Machines (in Russian). Theor Exp Electron 4, 1932.

[12] G. Nikolski, On Automatic Stability of a Ship on a Given Course (in Russian). Proc Central Commun Lab, 1934.

[13] Y. Z. Tzykpin, Theory of control relay systems (In russian). Goztekhizdat, 1955. 
[14] — Relay control systems. Cambbridge Press: UK, 1984.

[15] D. Anosov, On stability of equilibrium points of relay systems. Automation and remote control, 1959.

[16] L. Fridman and A. Levant, Sliding Mode Control In Engineering. Marcel Dekker, 2002, ch. Higher Order Sliding Modes.

[17] S. Emelyanov, S. Korovin, and L. Levantovskij, "Second order sliding modes in controlling uncertain systems," Soviet journal of computer and systems sciences, vol. 24, 1986.

[18] T. Sanchez and J. Moreno, "On a sign controller for the triple integrator," in Decision and Control (CDC), 2013 IEEE 52nd Annual Conference on, Dec 2013, pp. 3566-3571.

[19] F. Castaños and L. Fridman, "Dynamic switching surfaces for output sliding mode control: An $H_{\infty}$ approach," Automatica, vol. 47, no. 9, pp. 1957 - 1961, 2011.

[20] A. Aparicio, F. Castaños, and L. Fridman, "Output feedback sliding-mode control with unmatched disturbances, an iss approach," International Journal of Robust and Nonlinear Control, vol. 26, no. 18, pp. 4056-4071, 2016.

[21] T. Osuna, Y. Orlov, and L. T. Aguilar, "L2-gain tuning of variable structure siso systems of relative degree n," International Journal of Control, vol. 0, no. 0, pp. 1-22, 2017.

[22] R. A. DeCarlo, S. H. Zak, and G. P. Matthews, "Variable structure control of nonlinear multivariable systems: a tutorial," Proceedings of the IEEE, vol. 76, no. 3, pp. 212-232, Mar 1988.

[23] R. Horn and C. Johnson, Matrix Analysis. Cambridge University Press, 1990.

[24] W. H. Young, "On classes of summable functions and their fourier series," Proceedings of the Royal Society of London A: Mathematical, Physical and Engineering Sciences, vol. 87, no. 594, pp. 225-229, 1912.

[25] E. D. Sontag, "On the input-to-state stability property," Systems and Control Letters, vol. 24, pp. 351-359, 1995.

[26] Y. V. Orlov, Discontinuous Systems, Lyapunov Analysis and Robust Synthesis under Uncertainty Conditions. Springer-Verlag London, 2009.

[27] A. Filoppov, Differential Equations with Discontinuous Righthand Sides. Springer, 1988.

[28] F. Morgan, Geometric Measure Theory 3rd Edition. San Diego: Academic Press, 2000.

[29] E. Sontag, "Smooth stabilization implies coprime factorization," Automatic Control, IEEE Transactions on, vol. 34, no. 4, pp. 435-443, Apr 1989. 